\title{
The Stiffness of Virus Shell: Influenza Lipid Shell
}

\author{
M.R. Fauzi ${ }^{1}$ Nurfa Risha ${ }^{1}$ Gede Aris Gunadi ${ }^{1}$ Luh Putu Budi Yasmini1 ${ }^{1, *}$ \\ ${ }^{I}$ Physics Education Study Program, Universitas Pendidikan Ganesha, Singaraja, Bali \\ ${ }^{*}$ Corresponding author.Email: budi.yasmini@undiksha.ac.id
}

\begin{abstract}
Virus is an infectious agent with microscopic size, a protective shell containing capsomere with protein material called a capsid, and the addition of a shell containing fat called a lipid coat. The one characteristic of a virus is about the shell stiffness, so the virus shell can be assumed as a spring. The value of stiffness of the virus shell is different for various viruses, depending on intrinsic properties (modulus of elasticity) and extrinsic properties (radius and thickness of the virus shell).

In this paper, we analyze the value of stiffness of the virus shell by analytical and simulation. The analytical method is based on plate and shell theory and Michell solution. The other, the simulation is based on finite element analysis (FEA) method. For influenza lipid shell, we find that the stiffness value of the shell based on plate and shell theory is $0.0236 \frac{\mathrm{nN}}{\mathrm{nm}}$. Based on Michell solution, the stiffness value of the shell is $0.0225 \frac{\mathrm{nN}}{\mathrm{nm}}$. Also, the stiffness value of influenza lipid by FEA method is $0.0227 \pm 0.0002 \frac{\mathrm{nN}}{\mathrm{nm}}$. Generally, based on the graph that the stiffness value of influenza lipid shell by analytical and simulation is approaching same.
\end{abstract}

Keywords: virus shell, stiffness, Michell equation, plate \& shell theory, and FEA

\section{INTRODUCTION}

Virus is the agent infectious with microscopic size which increase they are amount in host cell [1]. Virus has two types, there is enveloped and non-enveloped virus. For example, the enveloped virus is Influenza virus, and the non-enveloped virus is Minute Virus of Mice (MVM). Enveloped of virus has important role in the first step to infection host cell [2].

The interesting part of virus is the shell, which the composition material is protein (this called capsid and sub-material capsid called capsomere) and fat (this called lipid). The shell is used to protect virus genomes, which consist of DNA or RNA only. The one characteristic of virus that can analyst in physics theories is stiffness of the virus shell which measured by Atomic Force Microscopy (AFM) [3].

In AFM method, virus assumed as ideal spring. The value of shell stiffness is known by determining the effective stiffness and cantilever stiffness. The stiffness of shell virus can be determined from theory of elasticity. Usually, the stiffness of the virus shell is determined by theory of plate and shell, firstly, we have to determine the bending energy and the stretching energy of the shell [4]. In this paper, we analyze a different way to know the stiffness of the virus shell by Michel equation.

In this paper, we analyze the value of stiffness of the virus shell by analytical and simulation. The analytical method is based on plate and shell theory and Michell solution. The simulation is based on finite element analysis (FEA) method. This paper explains how the solution of Michell equation can be useful to determine the stiffness of the virus shell too, beside of by plate and shell theory. We find the ratio of bending energy to stretching energy, and this ratio is related to the stiffness of the virus shell.

\section{STIFFNESS OF VIRUS SHELL}

\subsection{Plate and Shell Theory}

In this subsection, we will discuss shortly how to know the value of stiffness of virus shell by plate and shell theory. Theoretically, the stiffness value of virus shell by the plate and shell theory is the sum of bending energy and stretching energy. As shown in Figure 1., deflection $w$ variance respect with the distance $d$ [2], 


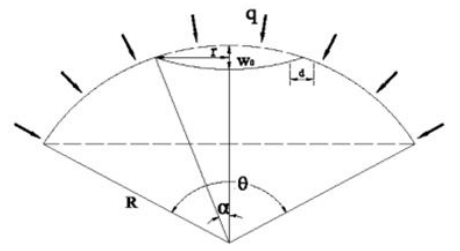

Figure 1. Buckling of the spherical shell

the value of stiffness of the virus shell based on plate and shell theory is

$\mathbf{k}=\frac{\mathbf{E h}^{2}}{\mathbf{R}}$,

where $R$ is the radius of the middle surface of the shell with $R=R_{1}+\frac{h}{2}$.

\subsection{Michell Solution}

In this subsection, we will analyze the stiffness of the virus shell Michell equation and thermodynamic pressure. Michell equation in terms of the radial external pressure is [5],

$p=\frac{h}{R}\left(\sigma_{\theta \theta}+\sigma_{\phi \phi}\right)$,

this term analog with Young-Laplace equation. The relation between thermodynamic pressure and 3D stress tensor of the virus shell from partition function of the virus shell is [6],

$p=-\frac{1}{4 \pi R^{2}}\left(\frac{\partial H}{\partial R}\right)_{N, T}=k_{B} T \frac{1}{4 \pi R^{2}} \frac{1}{Q}\left(\frac{\partial Q}{\partial R}\right)_{N, T}$.

$Q(N, V, T)=\frac{1}{\Lambda^{3 N} N !} \int d r_{1} \int d r_{2} \int d r_{N} e^{-\beta u\left(r_{i}\right)}$,

$p=-\left(\frac{\partial H}{\partial V}\right)_{N_{s} T}=k_{B} T\left(\frac{\partial}{\partial V} \ln Q\right)_{N_{s} T}$

were,

with $\beta=\frac{1}{k_{B} T}$.

In sphere coordinate system, Equation (3) can be written as,

Moreover, we discuss about position of capsomer. The capsomere position and the surface of the capsid showed in Figure 2. [7],

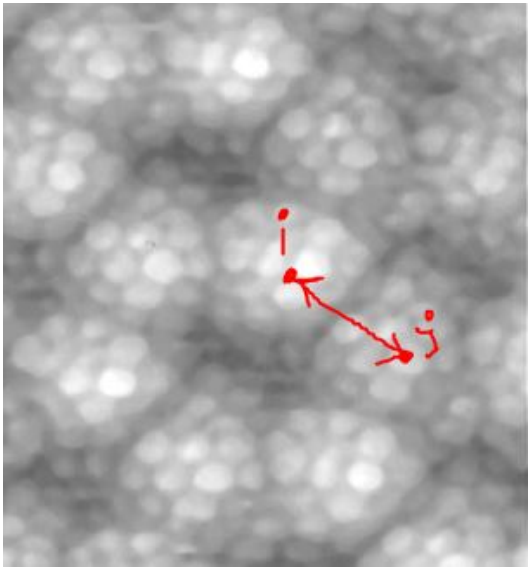

(a)

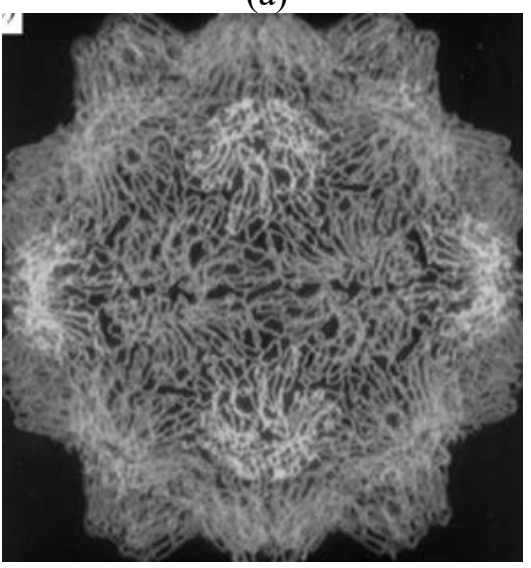

(b)

Figure 2. (a) Capsomere $i$ and $j$ in Turnip Yellow Mosaic Virus (TYMV) capsid and (b) TYMV capsid

The position of capsomer is written as ,

$\boldsymbol{r}_{i}=R \boldsymbol{t}_{i}$

where $R$ is radius of capsomer and $t$ çoordinate of $i^{\text {th }}$ capsomere as shown in Figure . Substituting Equation (6) to Equation (4) so we obtain that

$$
Q(N, V, T)=\frac{R^{3 N}}{\Lambda^{3 N} N !} \int d \boldsymbol{t}_{1} \int d \boldsymbol{t}_{2} \int d \boldsymbol{t}_{N} e^{-\beta u\left(R t_{i}\right)} .
$$

Next, the derivative of Equation (7) with respect to the radius of the capsid is

$\frac{\partial Q}{\partial R}=\frac{3 N Q}{R}-\beta \frac{\partial u}{\partial R} Q$,

where $u\left(r_{i}\right)=\frac{1}{2} \sum_{i, j} V\left(r_{i j}\right)$ describe the sum of all capsomere interaction, so

$\frac{\partial u}{\partial R}=\frac{1}{2}\left(\sum_{i, j} \frac{\partial V\left(r_{i j}\right)}{\partial r_{i j}} \frac{\partial r_{i j}}{\partial R}\right)$,

and 
$\frac{\partial r_{i j}}{\partial R}=\frac{1}{R} \frac{\left(\vec{r}_{i j} \cdot \hat{e}_{\alpha}\right)\left(\vec{r}_{i j} \cdot \hat{e}_{\alpha}\right)}{r_{i j}}$,

So, Equation (5) can written into

$p=k_{B} T\left(\frac{N}{V}-\frac{1}{3 V k_{B} T}\left(\frac{1}{2} \sum_{i, j} \frac{\partial V\left(r_{i j}\right)}{\partial r_{i j}} \frac{\left(\vec{r}_{i j}-\hat{e}_{a}\right)\left(\vec{r}_{i j} \cdot \hat{e}_{\beta}\right)}{r_{i j}}\right)\right)$,

and the definition of virial stress tensor 3D become [6],

$\sigma_{a \beta}^{3 D}=\frac{N k_{B} T}{V}-\frac{1}{V}\left(\frac{1}{2} \sum_{i, j} \frac{d V\left(r_{i j}\right)}{d r_{i j}} \frac{\left(\overrightarrow{r_{i j}} \cdot \hat{e}_{\alpha}\right)\left(\overrightarrow{r_{i j}} \cdot \hat{e}_{\beta}\right)}{r_{i j}}\right)$.

Based on Equation (11), the lateral and radial components of virial stress tensor in spherical coordinate [6] are,

$\sigma_{\theta \theta}^{3 D}=\frac{N k_{B} T}{V}-\frac{1}{V}\left(\frac{1}{2} \sum_{i, j} \frac{d V\left(r_{i j}\right)}{d r_{i j}} \frac{\left(\overrightarrow{r_{l j}} \cdot \hat{e}_{\theta}\right)^{2}}{r_{i j}}\right)$

$\sigma_{\phi \phi}^{3 D}=\frac{N k_{B} T}{V}-\frac{1}{V}\left(\frac{1}{2} \sum_{i, j} \frac{d V\left(r_{i j}\right)}{d r_{i j}} \frac{\left(\vec{r}_{i j} \cdot \hat{e}_{\phi}\right)^{2}}{r_{i j}}\right)$

$\sigma_{r r}^{3 D}=\frac{N k_{B} T}{V}-\frac{1}{V}\left(\frac{1}{2} \sum_{i, j} \frac{d V\left(r_{i j}\right)}{d r_{i j}} \frac{\left(\overrightarrow{r_{l j}} \cdot \hat{e}_{r}\right)^{2}}{r_{i j}}\right)$

Because of the sum of stress tensor 3D has relation with pressure, based on this equation

$\sigma_{\theta \theta}^{3 D}+\sigma_{\phi \phi}^{3 D}+\sigma_{r r}^{3 D}=3 p$,

so, the equation of the pressure in stress tensor is

$$
p=\frac{1}{3}\left(\sigma_{\theta \theta}^{3 D}+\sigma_{\phi \phi}^{3 D}+\sigma_{r r}^{3 D}\right)
$$

We can neglect the radial component because the stiffness of the capsid relates to lateral stress tensor, which plays a role in the interaction between each capsomere particle. Then Equation (3) can be written as in Equation (15) is called the Young-Laplace equation,

$p=\frac{1}{3}\left(\sigma_{\theta \theta}^{3 D}+\sigma_{\phi \phi}^{3 D}\right)=\frac{1}{3}\left(2 \sigma_{T}^{3 D}\right)$.

The relation between stress tensor $2 \mathrm{D}$ and $3 \mathrm{D}$ for tangential component-based virial stress tensor is [6],

$\Omega^{d} \sigma_{\alpha \beta}^{d}=-\frac{1}{2} \sum_{i, j} \frac{d V\left(r_{i j}\right)}{d r_{i j}} \frac{\left(\overrightarrow{r_{i j}} \cdot \hat{e}_{\alpha}\right)\left(\overrightarrow{r_{i j}} \cdot \hat{e}_{\beta}\right)}{r_{i j}}$, where $\Omega^{d}$ is the surface area for $d=2$ and the volume for $d=3$, so the equation of stress tensor $2 \mathrm{D}$ and $3 \mathrm{D}$ are

$$
\sigma_{\theta \theta}^{2 D}=-\frac{1}{4 \pi R^{2}} \frac{1}{2} \sum_{i, j} \frac{d V\left(r_{i j}\right)}{d r_{i j}} \frac{\left(\overrightarrow{r_{i j}} \cdot \hat{e}_{\theta}\right)^{2}}{r_{i j}}
$$

and

$$
\sigma_{\theta \theta}^{3 D}=-\frac{3}{4 \pi R^{3}} \frac{1}{2} \sum_{i, j} \frac{d V\left(r_{i j}\right)}{d r_{i j}} \frac{\left(\overrightarrow{r_{i j}} \cdot \hat{e}_{\theta}\right)^{2}}{r_{i j}} .
$$

The relation between stress tensor $2 \mathrm{D}$ and $3 \mathrm{D}$ is

$$
\sigma_{\theta \theta}^{3 D}=\frac{3}{R} \sigma_{\theta \theta}^{2 D},
$$

where $\sigma_{\theta \theta}^{2 D}=\sigma_{\phi \phi}^{2 D}$ and stress tensor at the surface of capsid is uniform. Then Equation (4) can rewrite as

$$
\begin{aligned}
& \sigma_{T}^{2 D}=-\frac{1}{4 \pi R^{2}} \frac{1}{2} \sum_{i, j} \frac{d V\left(r_{i j}\right)}{d r_{i j}} \frac{\left\{\left(\overrightarrow{r_{i j}} \cdot \hat{e}_{\theta}\right)^{2}+\left(\overrightarrow{r_{i j}} \cdot \hat{e}_{\phi}\right)^{2}\right\}}{2 r_{i j}} \\
& 2 \sigma_{T}^{2 D}=-\frac{1}{4 \pi R^{2}} \frac{1}{2} \sum_{i, j} \frac{d V\left(r_{i j}\right)}{d r_{i j}} \frac{\left\{\left(\overrightarrow{r_{i j}} \cdot \hat{e}_{\theta}\right)^{2}+\left(\overrightarrow{r_{l j}} \cdot \hat{e}_{\phi}\right)^{2}\right\}}{r_{i j}} \\
& 2 \sigma_{T}^{2 D}=\sigma_{\theta \theta}^{2 D}+\sigma_{\phi \phi}^{2 D}=2 \sigma_{\theta \theta}^{2 D} \\
& \sigma_{T}^{2 D}=\sigma_{\theta \theta}^{2 D} .
\end{aligned}
$$

Based on Equation (5), we obtain that

$p=\frac{2}{R} \sigma_{\theta \theta}^{2 D}$.

Next, determine the stiffness of capsid using Michell solution concerning the disc of the shell of virus in spherical coordinate as shown in Figure 3. [8].

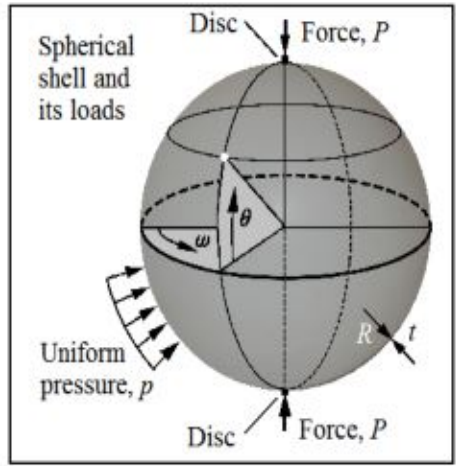

Figure 3. Spherical shell with external pressure $p$

In Figure 4., the white point is the disc of shell virus and stress tensor assumed that the shell is uniform, 


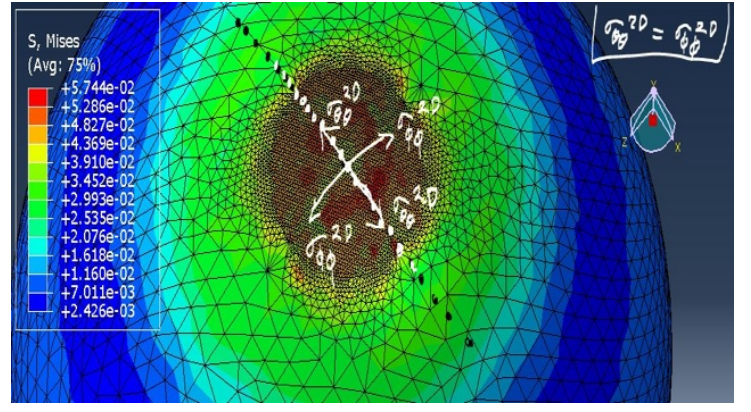

Figure 4. Shell of the virus drawn by FEA.

So, we can relate with stress tensor base Michell solution. Using stress tensor tangential component $(\theta)$ from Michell solution for the disc of virus shell,

$\sigma_{\theta \theta}^{2 D}=-\frac{a}{R^{2}}+b$

where $\sigma_{\theta \theta}^{2 D}$ is stress tensor 2D for component $\theta, R$ is the radius middle surface of virus shell, $a$, and $b$ is a constant. Substitute Equation (7) to Equation (6), we can write Equation (19) become,

$p=\frac{2}{R}\left(-\frac{a}{R^{2}}+b\right)$.

The Equation (8) is describing the pressure of the shell.

To find out the equation of stiffness of virus shell, we need to determine the pressure equation base plane stress theory. In elasticity theory, the general equation for plane stress is [5]

$\sigma_{\alpha \beta}=\frac{E}{\left(1-v^{2}\right)}\left(e_{\alpha \beta}(1-v)+v \delta_{\alpha \beta} e_{\gamma \gamma}\right)$,

where $E$ is the elasticity modulus of virus shell with unit force per area. When $\sigma_{\theta \theta}=\sigma_{\phi \phi}$ then $e_{\theta \theta}=e_{\phi \phi}$, so Equation (9) becomes

$\sigma_{\theta \theta}=\frac{E}{(1-v)} \frac{\delta R}{R}$,

with $e_{\theta \theta}=\frac{\delta R}{R}$. Then substitute Equation (10) to Equation (2), we obtain the equation of pressure $p$ based on elasticity theory,

$p=\frac{2 E}{(1-v)} \frac{h}{R^{2}} \delta R$.

The last step is to find out the stiffness of the virus shell by using Equation (11) and Equation (8),

$\frac{2}{R}\left(-\frac{a}{R^{2}}+b\right)=\frac{2 E}{(1-v)} \frac{h}{R^{2}} \delta R$

$$
\frac{-a+b R^{2}}{R}=\frac{E}{(1-v)} h \delta R .
$$

Next step is determining the constants $a$ and $b$ with boundary condition $\sigma_{r r}\left(R_{2}\right)=-\sigma$ and $\sigma_{r r}\left(R_{1}\right)=0$, we obtain that

$a=\frac{R_{1}^{2} R_{2}^{2}}{\left(R_{2}^{2}-R_{1}^{2}\right)} \sigma$

and

$b=-\frac{R_{2}^{2}}{\left(R_{2}^{2}-R_{1}^{2}\right)} \sigma$,

where $\sigma$ is external load constant. So, Equation (12) can written as,

$$
\sigma=-\frac{E h}{(1-v)} \frac{\left(R_{2}^{2}-R_{1}^{2}\right)}{\left(R_{1}^{2}+R^{2}\right)} \frac{R}{R_{2}^{2}} \delta R .
$$

Buckling at shell happens while radial load increase until the certain limit, then the shape of virus shell changes from the equilibrium shape. The external load at virus shell until buckling happens is

$$
\sigma=\frac{F}{2 r}=\frac{F}{2 R \sin \alpha} \approx \frac{F}{2 R \alpha},
$$

where $\alpha$ is the angle buckling of the virus shell. Then substitute Equation (14) to Equation (13), we obtain

$$
\frac{F}{\delta R}=-\frac{2 \alpha E h}{(1-v)} \frac{\left(R_{2}^{2}-R_{1}^{2}\right)}{\left(R_{1}^{2}+R^{2}\right)} \frac{R^{2}}{R_{2}^{2}} .
$$

In the thin shell model, the value of $R \approx R_{1}$, so Equation (15) become

$$
\frac{F}{\delta R}=-\frac{\alpha E h}{(1-v)} \frac{\left(R_{2}^{2}-R_{1}^{2}\right)}{R_{2}^{2}} .
$$

The relation between external radius and internal radius is

$R_{1}=R_{2}-h$

and substitute to Equation (16), we get

$\frac{F}{\delta R}=-\left\{\frac{2 \alpha}{(1-v)}\right\} \frac{E h^{2}}{R_{2}}$,

Based on the Hooke's equation,

$\frac{F}{\delta R}=-k$,

then the stiffness of the virus shell is 
$k=\left\{\frac{2 \alpha}{(1-v)}\right\} \frac{E h^{2}}{R_{2}}$

the constant of $\frac{2 \alpha}{(1-v)}$ can be indicated with $c$, so Equation (20) can written as,

$k=c \frac{E h^{2}}{R_{2}}$,

By relating the equation of critical pressure in spherical shell $[4,10]$,

$p_{\text {critical }}=\frac{2}{\sqrt{3\left(1-v^{2}\right)}} \frac{E h^{2}}{R^{2}}$,

then we get $c$ is $\frac{2}{\sqrt{3\left(1-v^{2}\right)}}$, so the stiffness is

$k=\frac{2}{\sqrt{3\left(1-v^{2}\right)}} \frac{E h^{2}}{R_{2}}$.

If the constant of $\frac{2}{\sqrt{3\left(1-v^{2}\right)}} \approx 1$ with range

$0 \leq v \leq 0.5$, so the value of equation $c$ is

$c \approx 1$.

then the equation of stiffness virus shell is

$k=\frac{E h^{2}}{R_{2}}$.

\section{INFLUENZA LIPID SHELL}

In this subsection, we will be discussed about the value of stiffness of a virus. The analyses are based on plate and shell theory, Michell solution, and finite element analysis. Finite Element Analysis (FEA) is a computerized method to predict behavior of something like the virus shall be affected by physical effects such as fatigue. In this model, the virus's shell is indented with a rigid hemisphere, and its radius is $10 \mathrm{~nm}$ for all models.

Influenza lipid shell have thickness $h=5 \mathrm{~nm}$, with the radius $R_{1}=45 \mathrm{~nm}, R=47.5 \mathrm{~nm}$ and $R_{2}=50 \mathrm{~nm}$, the Poisson ratio $v=0.4$, and the value of modulus elasticity $E=0.045 \frac{n N}{n m^{2}}[11,12]$. By FEA method, the model of influenza lipid shell after indented until 10 $\mathrm{nm}$ is shown in Figure 5.,

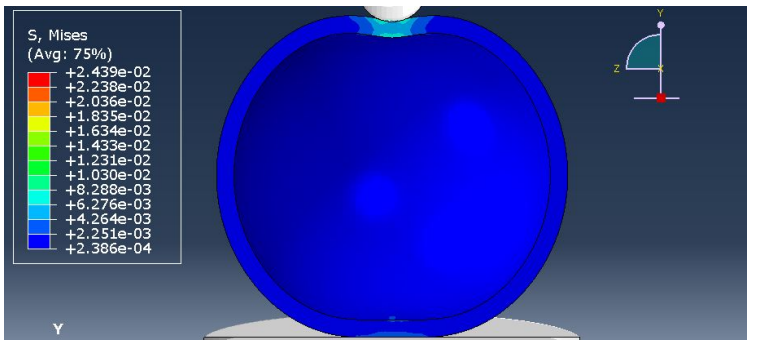

Figure 5. Influenza lipid by FEA method

Based on the characteristic of this shell, the stiffness value of the shell based on plate and shell theory is $k_{\text {lipid }}=\frac{E h^{2}}{R}=\frac{(0.045 \mathrm{GPa})(5.0 \mathrm{~nm})^{2}}{(47.5 \mathrm{~nm})}=0.0236 \frac{\mathrm{nN}}{\mathrm{nm}}=0.024 \frac{\mathrm{nN}}{\mathrm{nm}}$,

and based on Michell solution is

$k_{\text {lipid }}=\frac{E h^{2}}{R_{2}}=\frac{(0.045 \mathrm{GPa})(5.0 \mathrm{~nm})^{2}}{(50 \mathrm{~nm})}=0.0225 \frac{\mathrm{nN}}{\mathrm{nm}} \approx 0.023 \frac{\mathrm{nN}}{\mathrm{nm}}$,

and the result of the FEA method shown in Figure 6.,

\section{FEA Method for lipid influenza}

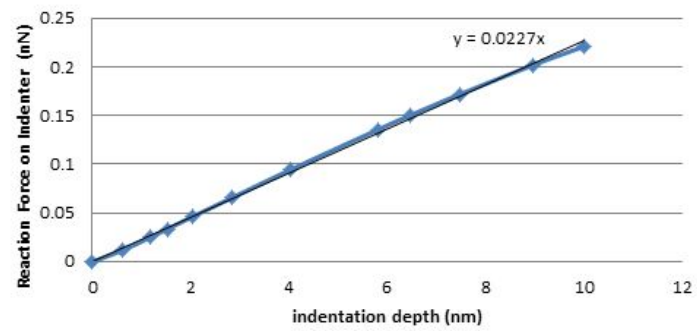

Figure 6. Gradient of lipid influenza with FEA method

The result of the FEA method gives the stiffness value of influenza lipid is 0.0227 with uncertainties of 0.0002 . So, the stiffness value of influenza lipid is $0.0227 \pm$ $0.0002 \frac{n N}{n m}$. The ratio of stiffness value between theory and numerically shown in Figure 7.,

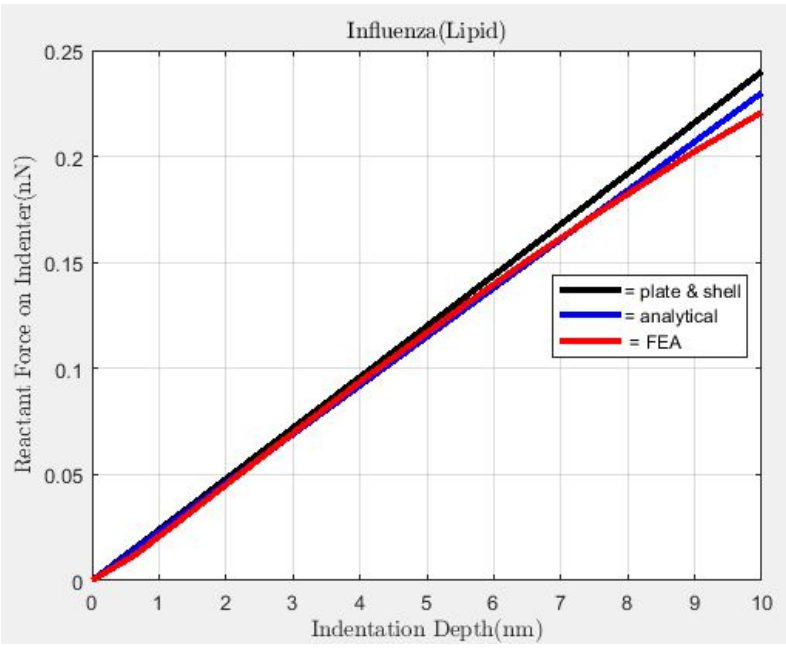

Figure 7. Plot of the stiffness value of influenza lipid for theory and numeric methods

Figure 7. has shown the relation between reaction force on indenter and indentation depth at depth 0 until 10 
nm. Generally, based on the graph that the stiffness value of influenza lipid shell by analytical and simulation is approaching same. The FEA result approaches analytical results (by Michell solution) at depth $2 \mathrm{~nm}$ until $8.9 \mathrm{~nm}$.

\section{CONCLUSIONS}

The virus is an infectious agent with microscopic size, a protective shell containing capsomere with protein material called a capsid, and the addition shell containing fat called a lipid coat. The one characteristic of a virus is about the shell stiffness. The value of stiffness of the virus shell is different for various viruses depending on intrinsic properties (modulus of elasticity) and extrinsic properties (radius and thickness of the virus shell).

In this paper, we analyze the value of stiffness of the virus shell by analytical and simulation. The analytical method is based on plate and shell theory and Michell solution. The other, the simulation is based on finite element analysis (FEA) method. For influenza lipid shell, we find that the stiffness value of the shell based on plate and shell theory is $0.0236 \frac{\mathrm{nN}}{\mathrm{nm}}$. Based on Michell solution, the stiffness value of the shell is $0.0225 \frac{\mathrm{nN}}{\mathrm{nm}}$. Also, the stiffness value of influenza lipid by FEA method is $0.0227 \pm 0.0002 \frac{n N}{n m}$. Generally, based on the graph that the stiffness value of influenza lipid shell by analytical and simulation is approaching same.

\section{ACKNOWLEDGMENTS}

We want to say thank you to Universitas Pendidikan Ganesha to support this research by Penelitian Dasar grant with contract number: 322/UN48.16/LT/2021.

\section{REFERENCES}

[1] H. R. Gelderblom, "Structure and Classification of Viruses," Med. Microbiol., no. May, 1996.

[2] B. Eshaghi et al., "Stiffness of HIV-1 Mimicking Polymer Nanoparticles Modulates Ganglioside-Mediated Cellular Uptake and Trafficking," vol. 2000649, pp. 1-13, 2020, doi: 10.1002/advs.202000649.

[3] M. G. Mateu, "Mechanical properties of viruses analyzed by atomic force microscopy: A virological perspective," Virus Res., vol. 168, no. 1-2, pp. 1-22, 2012, doi: 10.1016/j.virusres.2012.06.008.

[4] J. M. Timoshenko, Stephen P. dan Gere, Theory of Elastic Stability, 2nd ed. New York: Mc-
Graw-Hill, 1961.

[5] L. . dan Landau and E. . Lifshitz, Theory of Elasticity. New York: Pergamon, 1986.

[6] R. Zandi and D. Reguera, "Mechanical properties of viral capsids," Phys. Rev. E - Stat. Nonlinear, Soft Matter Phys., vol. 72, no. 2, 2005, doi: 10.1103/PhysRevE.72.021917.

[7] A. J. Malkin, Y. G. Kuznetsov, and A. Mcpherson, "Viral capsomere structure, surface processes and growth kinetics in the crystallization of macromolecular crystals visualized by in situ atomic force microscopy," vol. 232, pp. 173-183, 2001.

[8] J. W. Hutchinson and J. M. T. Thompson, "Imperfections and energy barriers in shell buckling," Int. J. Solids Struct., vol. 148-149, no. September 2017, pp. 157-168, 2018, doi: 10.1016/j.ijsolstr.2018.01.030.

[9] P. N. Khakina, "Buckling Load of Thin Spherical Shells Based on the Theorem of Work and Energy," Int. J. Eng. Technol., vol. 5, no. 3, pp. 392-394, 2013, doi: 10.7763/ijet.2013.v5.581.

[10] A. Evkin, M. Kolesnikov, and D. A. Prikazchikov, "Buckling of a spherical shell under external pressure and inward concentrated load: Asymptotic solution," Math. Mech. Solids, vol. 22, no. 6, pp. 1425-1437, 2017, doi: $10.1177 / 1081286516635872$.

[11] A. Ahadi, D. Johansson, and A. Evilevitch, "Modeling and simulation of the mechanical response from nanoindentation test of DNAfilled viral capsids," pp. 183-199, 2013, doi: 10.1007/s10867-013-9297-9.

[12] M. G. Mateu, "Mechanical properties of viruses analyzed by atomic force microscopy: A virological perspective," vol. 168, pp. 1-22, 2012.

[13] N. Kol et al., "A Stiffness Switch in Human Immunodeficiency Virus," vol. 92, no. March, 2007, doi: 10.1529/biophysj.106.093914.

[14] E. Jiménez-Piqué, L. Llanes, and M. Anglada, "Resistance to Contact Deformation and Damage of Hard Ceramics," Compr. Hard Mater., vol. 2, pp. 367-383, 2014, doi: 10.1016/B978-0-08-096527-7.00032-5. 